OPEN ACCESS

Edited by: Alessandro Gozzi,

Italian Institute of Technology (IIT), Italy

Reviewed by:

Hannes Almgren,

Ghent University, Belgium

Shella Keilholz,

Emory University, United States

Aldo Cordova-Palomera,

Stanford University, United States

*Correspondence:

Vince D. Calhoun

vcalhoun@gsu.edu

Mohammad S. E. Send

mseslampanah@gatech.edu

Received: 10 August 2020 Accepted: 15 December 2020

Published: 21 January 2021

Citation:

Sendi MSE, Zendehrouh E,

Miller RL, Fu Z, DU Y, LiU J,

Mormino $E C$, Salat $D H$ and Calhoun VD (2021) Alzheimer's

Disease Projection From Normal

to Mild Dementia Reflected in Functional Network Connectivity:

A Longitudinal Study.

Front. Neural Circuits 14:593263.

doi: 10.3389/fncir.2020.593263

\section{Alzheimer's Disease Projection From Normal to Mild Dementia Reflected in Functional Network Connectivity: A Longitudinal Study}

\author{
Mohammad S. E. Sendi 1,2,3*, Elaheh Zendehrouh4, Robyn L. Miller,3, Zening Fü, \\ Yuhui Du3,5, Jingyu Liu'3,4, Elizabeth C. Mormino ${ }^{6,7}$, David H. Salat ${ }^{8,9}$ and \\ Vince D. Calhoun ${ }^{1,2,3,4 *}$ \\ ${ }^{1}$ Wallace H. Coulter Department of Biomedical Engineering at Georgia Institute of Technology and Emory University, Atlanta, \\ GA, United States, ${ }^{2}$ School of Electrical and Computer Engineering, Georgia Institute of Technology, Atlanta, GA, \\ United States, ${ }^{3}$ Tri-Institutional Center for Translational Research in Neuroimaging and Data Science, Georgia State \\ University, Georgia Institute of Technology, Emory University, Atlanta, GA, United States, ${ }^{4}$ Department of Computer Science, \\ Georgia State University, Atlanta, GA, United States, ${ }^{5}$ School of Computer and Information Technology, Shanxi University, \\ Taiyuan, China, ${ }^{6}$ School of Medicine, Stanford University, Palo Alto, CA, United States, ${ }^{7}$ Department of Neurology \\ and Neurological Sciences, School of Medicine, Stanford University, Stanford, CA, United States, ${ }^{8}$ Harvard Medical School, \\ Cambridge, MA, United States, ${ }^{9}$ Massachusetts General Hospital, Boston, MA, United States
}

Background: Alzheimer's disease (AD) is the most common age-related problem and progresses in different stages, including mild cognitive impairment (early stage), mild dementia (middle-stage), and severe dementia (late-stage). Recent studies showed changes in functional network connectivity obtained from resting-state functional magnetic resonance imaging ( $\mathrm{rs}-\mathrm{fMRI}$ ) during the transition from healthy aging to $A D$. By assuming that the brain interaction is static during the scanning time, most prior studies are focused on static functional or functional network connectivity (sFNC). Dynamic functional network connectivity (dFNC) explores temporal patterns of functional connectivity and provides additional information to its static counterpart.

Method: We used longitudinal rs-fMRl from 1385 scans (from 910 subjects) at different stages of $A D$ (from normal to very mild $A D$ or vmAD). We used group-independent component analysis (group-ICA) and extracted 53 maximally independent components (ICs) for the whole brain. Next, we used a sliding-window approach to estimate dFNC from the extracted $53 \mathrm{ICs}$, then group them into 3 different brain states using a clustering method. Then, we estimated a hidden Markov model (HMM) and the occupancy rate $(\mathrm{OCR})$ for each subject. Finally, we investigated the link between the clinical rate of each subject with state-specific FNC, OCR, and HMM.

Results: All states showed significant disruption during progression normal brain to vmAD one. Specifically, we found that subcortical network, auditory network, visual network, sensorimotor network, and cerebellar network connectivity decrease in vmAD 
compared with those of a healthy brain. We also found reorganized patterns (i.e., both increases and decreases) in the cognitive control network and default mode network connectivity by progression from normal to mild dementia. Similarly, we found a reorganized pattern of between-network connectivity when the brain transits from normal to mild dementia. However, the connectivity between visual and sensorimotor network connectivity decreases in vmAD compared with that of a healthy brain. Finally, we found a normal brain spends more time in a state with higher connectivity between visual and sensorimotor networks.

Conclusion: Our results showed the temporal and spatial pattern of whole-brain FNC differentiates $A D$ form healthy control and suggested substantial disruptions across multiple dynamic states. In more detail, our results suggested that the sensory network is affected more than other brain network, and default mode network is one of the last brain networks get affected by AD In addition, abnormal patterns of whole-brain dFNC were identified in the early stage of $A D$, and some abnormalities were correlated with the clinical score.

Keywords: Alzheimer's disease, resting state fMR imaging, hidden Markov model, longitudinal study, dynamic functional network connectivity

\section{INTRODUCTION}

Alzheimer's disease $(\mathrm{AD})$ is the most common age-related dementia, typically affecting individuals over 65 years of age (Masters et al., 2015). AD usually progresses slowly in several stages, including mild (early stage), moderate (middle stage), and severe (late stage) (Ryan and Rossor, 2011). To date, there is no way to cure AD, but some medications can decelerate its progress (Yiannopoulou and Papageorgiou, 2020). Therefore, predicting the progression from a normal stage to mild cognitive impairment and further to $\mathrm{AD}$ itself is an important step toward early medical intervention.

Resting-state functional magnetic resonance imaging (rsfMRI) that indirectly measures neural processing in the brain based on the blood oxygenation can be used to identify spatially distributed networks in the brain. In recent years, functional connectivity or its network analog functional network connectivity (FNC), including dynamic (dFC/dFNC) and static $(\mathrm{sFC} / \mathrm{sFNC})$, achieved from rs-fMRI time series has uncovered a great deal of knowledge about the brain dysconnectivity in various neurological disorder including schizophrenia (Abrol et al., 2017; Sendi et al., 2020), major depression disorder (Zhi et al., 2018), autism (Cerliani et al., 2015; de Lacy et al., 2017; Mash et al., 2019), ADHD (Wang et al., 2018), and AD (Brier et al., 2014). In particular for $\mathrm{AD}$, previous studies reported a reduction in the default-mode network FC in $\mathrm{AD}$ compared with mild cognitive impairment (MCI) patients and healthy subjects (Soman et al., 2020). Another study reported a difference in the FC of sensorimotor network (SMN), visual network (VSN), and default mode network of healthy control (HC) subjects and $\mathrm{AD}$ patients (Zheng et al., 2017).

By assuming that FNC is invariant, or static over time, many of the AD-related studies mentioned above have focused on
sFC/sFNC and ignored dFC/dFNC. Indeed, unlike conventional static functional network connectivity (sFNC), which is obtained from the correlation within an entire time series, dFNC refers to the connectivity between any pair of brain networks within sub-intervals of time series (Calhoun et al., 2014). In fact, dFNC research suggests that cognitive deficits and clinical symptoms associated with many neurological disorders do not only depend on the strength of the connectivity between any pair of brain regions but also on the variation of connectivity strength of those regions over time (Calhoun et al., 2014; Damaraju et al., 2014; Zhi et al., 2018; Zendehrouh et al., 2020). In recent years, a few papers studied dFNC in AD. For instance, we investigated whole-brain $\mathrm{dFNC}$ in $\mathrm{AD}$ and subcortical ischemic vascular disease (SIVD) (Fu et al., 2019). Another study explored the temporal properties of dFNC associated with dementia in Parkinson's disease (Fiorenzato et al., 2019). However, the longitudinal dFNC changes from cognitive normal to mildly then severely cognitively impaired has not been extensively explored.

In the current study, we explored the temporal dynamics of the whole-brain FNC from 1385 rs-fMRI scans of HC and very mild $\mathrm{AD}(\mathrm{vmAD})$. We used a sliding window approach followed by the k-means clustering method to identify a set of connectivity states (Calhoun et al., 2014). Next, we calculated between-state transition probability via hidden Markov model (HMM) and the amount of the time each subject spends in a state, called occupancy rate or OCR, to model the temporal properties of dFNC. We investigated the correlation between HMM and OCR features with the clinical dementia rating scale sum of boxes (CDR-SOB) scores. In addition, we explored the link between state-specific connectivity features with CDR-SOB. Finally, we trained a support vector machine (SVM) to predict from HC to vmAD based on the sFNC connectivity features and dFNC features, including HMM and OCR. 


\section{MATERIALS AND METHODS}

\section{Participants}

In this study, the data we used are from the longitudinal Open Access Series of Imaging Studies (OASIS)-3 cohort, which was collected from several ongoing studies in the Washington University Knight Alzheimer Disease Research Center over 15 years (LaMontagne et al., 2019). This data contains 1385 rsfMRI imaging and related clinical and demographic data at the time of scanning (from 910 subjects) with age ranging from 42 to 95 years. For each subject, the imaging data, demographic, and clinical dementia rating (CDR) scale were used in any stage of cognitive functionality. All participant must have CDR $\leq 1$ at the time of the clinical core assessment and once the participant reached $\mathrm{CDR}=2$ or $\mathrm{CDR}-\mathrm{SOB}>9$, they were no longer eligible for the study (LaMontagne et al., 2019). We evaluated the cognitive stage of the participants at the time of the scanning based on the CDR-SOB scores and organized them in 2 groups, including healthy control or $\mathrm{HC}(\mathrm{CDR}-\mathrm{SOB}=0)$, very mild $\mathrm{AD}$ or vmAD $(0.5 \leq \mathrm{CDR}-\mathrm{SOB} \leq 9)$ (O'Bryant et al., 2008). In total, we have 1028 scan of HC, 357 scans of vmAD patients. The demographic information is provided in Table $\mathbf{1}$.

\section{Imaging Data Acquisition}

Two Trio 3T with a 20-channel head coil was used to collect imaging data (Siemens Medical Solutions United States, Inc). High resolution $\mathrm{T} 2{ }^{*}$-weighted functional images were obtained by an echoplanar imaging or EP sequence with $\mathrm{TE}=27 \mathrm{~ms}$, $\mathrm{TR}=2.2 \mathrm{~s}$, flip angle $=90^{\circ}$, slice thickness $=4 \mathrm{~mm}$, slice gap $=4 \mathrm{~mm}$, matrix size $=64$, and voxel size of $1 \mathrm{~mm} \times 1 \mathrm{~mm} \times 1.25 \mathrm{~mm}$. The duration of the scanning was 6 min.

\section{Data Preprocessing}

The analytic pipeline used in this study is shown in Figure $\mathbf{1}$. The following subsections describe the detail of this pipeline. In the first step (Step 1 in Figure 1), the first five dummy scans were discarded before preprocessing. We used statistical parametric mapping $\left(\mathrm{SPM} 12^{1}\right)$ default slice timing routines to correct differences in image acquisition time between slices. The

${ }^{1}$ https://www.fil.ion.ucl.ac.uk/spm/

TABLE 1 | Demographic and clinical information.

\begin{tabular}{llc}
\hline & \multicolumn{1}{c}{$N$} & 1028 \\
HC & Gender (M/F) & $415 / 613$ \\
& Age & $69.83 \pm 8.64$ \\
& CDR-SOB & $0 \pm 0$ \\
\hline & $N$ & 357 \\
vmAD & Gender (M/F) & $215 / 142$ \\
& Age & $75.10 \pm 7.85$ \\
CDR-SOB & $2.68 \pm 2.10$ \\
\hline SC, healthy control; vmAD, very mild Alzheimer Disease; M, male; F, female; CDR-
\end{tabular}

reference slice was chosen as the slice acquired in the middle of the sequence. Rigid body motion correction was then applied to account for subject head movement, with 3-dimensional brain translations and 3-dimensional rotations estimated. Next, the imaging data underwent spatial normalization to the standard Montreal Neurological Institute (MNI) space using the echoplanar imaging (EPI) template and the default bounding box provided by the SPM toolbox and was resampled to $3 \times 3 \times 3 \mathrm{~mm}^{3}$. Finally, a Gaussian kernel with a full width at half maximum (FWHM) of $6 \mathrm{~mm}$ was used to smooth the fMRI images.

In this study, a set of robust network priors were used to extracted comparable components across subjects from the OASIS dataset. The network priors were extracted via the NeuroMark pipeline ( $\mathrm{Du}$ et al., 2020; Fu et al., 2020, 2021). This framework performed group ICA with model order as 100 on two healthy controls datasets, human connectome project $\left(\mathrm{HCP}^{2}, 823\right.$ subjects after the subject selection) and genomics superstruct project (GSP ${ }^{3}, 1005$ subjects after the subject selection) for creating the network priors. The extracted independent components (ICs) from the two datasets were matched by comparing the corresponding grouplevel spatial maps. If they show a higher spatial correlation than a given threshold $(=0.4)$, we consider that the IC pairs were reproducible. The reproducible ICs pairs were further evaluated by examining their spatial activations and lowfrequency fluctuations of their corresponding time-courses (TCs). 53 pairs of ICs were identified as meaningful and reproducible, arranging into 7 functional domains based on their anatomic and functional prior knowledge. These ICNs included subcortical network (SCN), auditory network (ADN), sensorimotor network (SMN), visual network (VSN), cognitive control network (CCN), default-mode network (DMN), and cerebellar network (CBN). The less noisy ICNs captured from the GSP dataset (Note that there were 53 ICNs from HCP which had similar spatial patterns) were chosen as the spatial network priors to back-reconstruct spatial maps and TCs for each subject. Also, to remove the remaining noise and artifact, the following post-processing procedures were performed on the time-courses signal before calculating the dynamic functional network connectivity (dFNC) between timecourses of ICs: (1) detrending linear, quadratic, and cubic trends; (2) conducting multiple regressions of the 6 realignment parameters and their temporal derivatives; (3) despiking detected outliers; and (4) low-pass filtering with a cut-off frequency of $0.15 \mathrm{~Hz}$. Figure 2 shows these seven domains. Also, Table 2 shows all 53 ICNs extracted by the NeuroMark pipeline in this study.

\section{Functional Network Connectivity}

The sFNC of each subject was calculated by computing the Pearson correlation between any pair of ICNs time series. With 53 ICNs, it resulted in 1378 whole-brain correlation values for

${ }^{2}$ https://www.humanconnectome.org/study/hcp-young-adult/document/1200subjects-data-release

${ }^{3}$ https://dataverse.harvard.edu/dataverse/GSP 


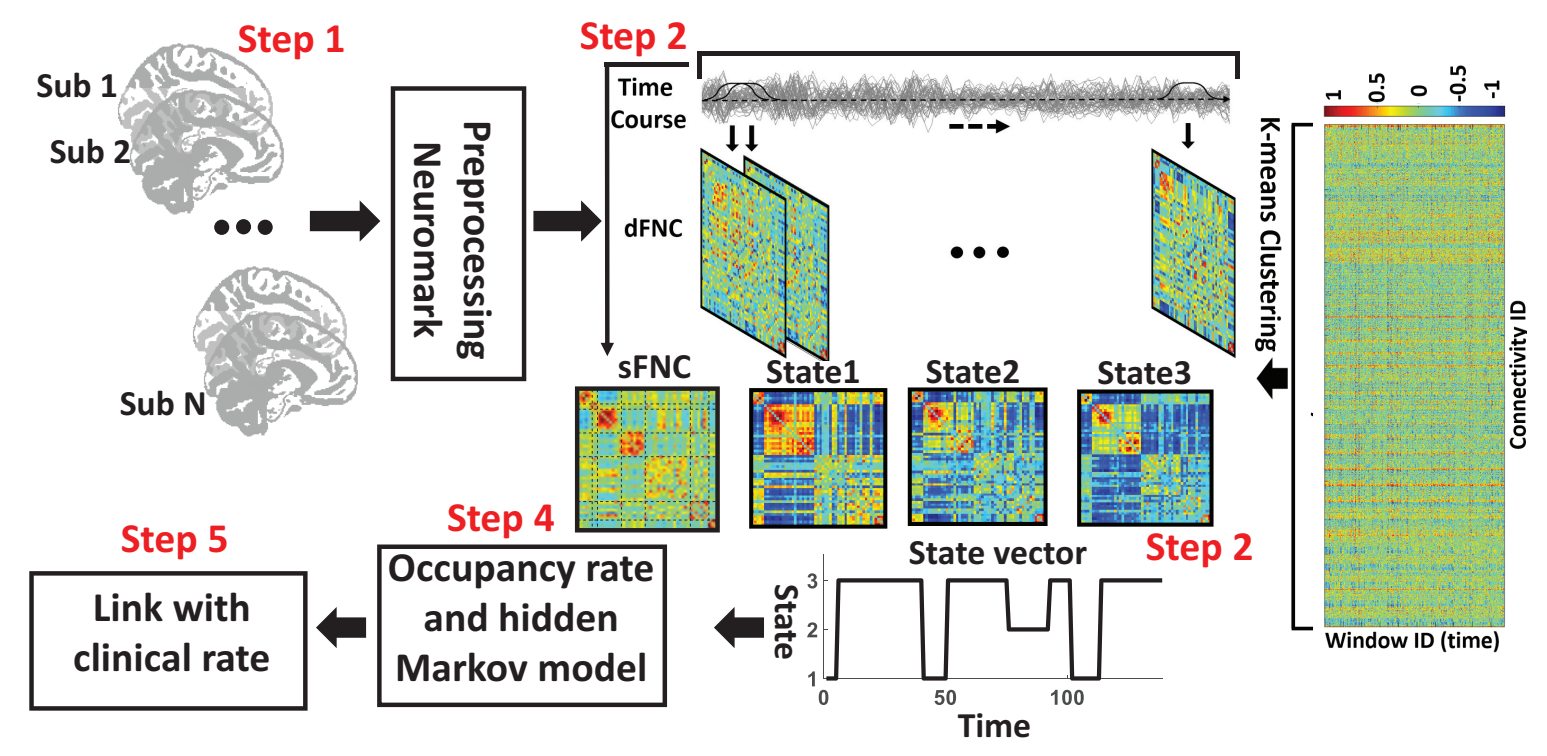

FIGURE 1 | Analytic pipeline. Step1: The time-course signal of 53 ICNs have been identified using group-ICA in the Neuromak template. Step2: After identifying 53 ICNs, a taper sliding window was used to segment the time-course signals and then calculated the functional network connectivity (FNC). Each subject has 139 FNCs with a size of $53 \times 53$. Also, we calculated static FNC for the entire time of recording. Step3: After vectorizing the FNC matrixes, we have concatenated them, and then a k-means clustering with correlation as distance metrics was used to group FNCs to three distinct clusters. Step4: Then, based on the state vector, we calculated between-state transition probability or hidden Markov model (HMM) features and occupancy rate (OCR) for each subject. In total, nine HMM features and three OCR were estimated from the state vector of each subject. Step5: To find a link between FNC features, including sFNC and dFNC feature with clinical dementia rating scale sum of boxes (CDR-SOB), we used partial correlation by accounting for age, gender.

each subject. In addition, for each subject $\mathrm{i}=1 \ldots \mathrm{N}$, the dynamic FNC (dFNC) of the whole brain was estimated via a sliding window approach, as shown in Figure 1. A tapered window obtained by convolving a rectangle (window size $=20$ TRs $=44 \mathrm{~s})$ with a Gaussian $(\sigma=3)$ was used to localize the dataset at each time point. It is worth mentioning that previous studies suggested that a window size between 30 and $60 \mathrm{~s}$ is a reasonable choice for capturing the dFNC fluctuation (Preti et al., 2017). Based on this past work, we used the $44 \mathrm{~s}$ as the window size. A covariance matrix, based on Pearson correlation, was calculated to measure the dFNC between ICs. The dFNC estimates of each window for each subject were concatenated to form a $(\mathrm{C} \times \mathrm{C} \times \mathrm{T})$ array (where $\mathrm{C}=53$ denotes the number of ICNs and $T=139$ ), which represented the changes in brain connectivity between ICNs as a function of time (Step 2 in Figure 1; Calhoun et al., 2014).

\section{Clustering and dFNC Latent Features}

We used k-means clustering to partition dFNC window into a set of separated clusters (states). Based on the elbow criterion (the ratio of within to between cluster distance), we found that the optimal number of clusters (i.e., $\mathrm{k}$ ) is 3 . We used correlation based on Pearson correlation as a distance metric in the clustering algorithm in 1000 iterations (Allen et al., 2014; Calhoun et al., 2014) (Step 3 in Figure 1). The output of this step is 3 states for all subjects and subject-specific state vector. The state vector shows that the state of the whole-brain FNC of each subject at a specific time. In the next step, we calculated the between-state transition probability based on HMM. The transition probability, $\mathrm{a}_{i j}$, is the probability of the system to transition from state $\mathrm{j}$ at time $\mathrm{t}$ to state $\mathrm{i}$ at time $\mathrm{t}+1$.

$$
a_{i j}=p(s(t+1)=i \mid s(t)=j)
$$

In addition, we computed the OCR of dFNCs in each state (Step 4 in Figure 1). In addition, for each subject, we averaged all dFNC belongs to a state as her/his state-specific FNC. In more detail, each subject has multiple dFNC in each state. Then, in each state, we used the average of dFNC (i.e., the average of 1378 connectivity features) of each subject as her/his statespecific FNC.

\section{Statistical Analysis}

To assess the link between dFNC features, including statespecific FNC, OCR, and HMM with CDR-SOB, we used partial correlation by accounting for age and gender. We performed statistical analysis on all 1378 whole-brain connectivity features, 9 HMM features, and 3 OCR features, separately (Step 5 in Figure 1). All $p$ values have been adjusted by the Benjamini-Hochberg correction method for multiple comparisons (Benjamini and Hochberg, 1995). The number of null hypothesis in state-specific FNC, OCR, and HMM were 1378,3 , and 9 , respectively.

\section{Dementia Progression Is Associated With Functional Network Connectivity}

In the next step, we explored whether functional network connectivity, including sFNC and dFNC features, can predict 


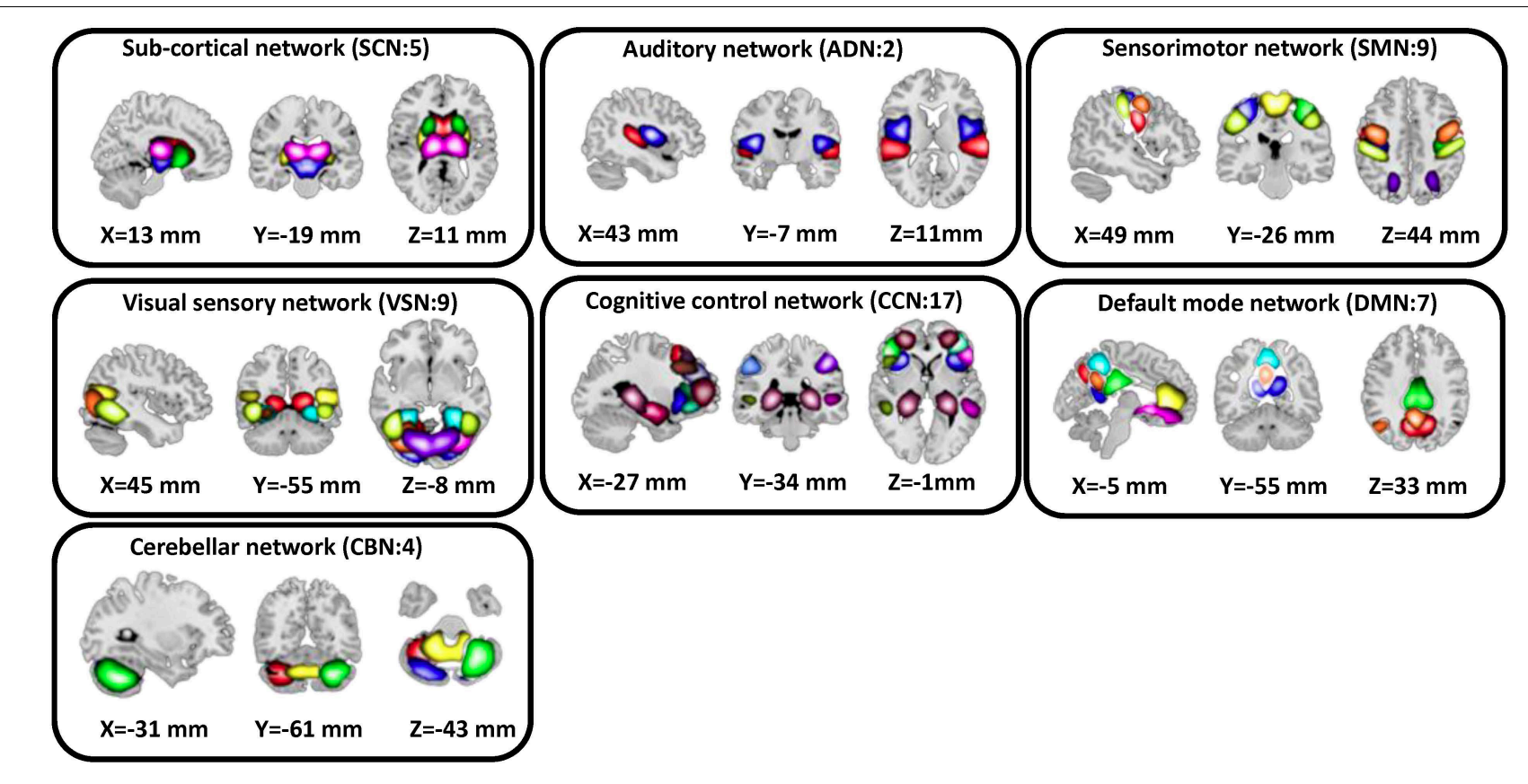

FIGURE 2 | In this study, we adopted the NeuroMark pipeline to extract reliable intrinsic connectivity networks (ICNs, in total, 53 components) that are replicated across independent datasets. All 53 independent components identified by group-ICA in the Neuromark template. We put them in seven networks, including subcortical network (SCN), auditory network (AND), visual sensory network (VSN), sensorimotor network (SMN), cognitive control network (CCN), default mode network (DMN), and cerebellar network (CBN).

the progression of $\mathrm{AD}$. We put subjects into two different groups. The first group contains those subjects who remained in $\mathrm{HC}$, whom we call them unconverted $\mathrm{HC}$ or uc-HC stage within the next 5 years of the first scan, and the second group contains those subjects which their cognitive functionality changed from $\mathrm{HC}$ to vmAD $(0.5 \leq \mathrm{CDR}-\mathrm{SOB} \leq 9)$, and we call c-HC. The first group contains 85 subjects (48 females and 37 males) with the mean age at 74.6478 and range between 65 and 85 . The second group contains 40 subjects (18 females and 22 males) in which the mean age is 74.6878 , and the range of the age is between 64 and 85 . We did not observe a significant difference between the age and gender of these two groups (age: Cohen's $\mathrm{d}=0.16$, two-sample $t$ test $t(123)=-0.90, p=0.36$; gender: Cohen's $\mathrm{d}=0.22$, twosample $t$ test $t(123)=1.19, p=0.23)$. We trained a SVM based on sFNC, OCR, and HMM features from the baseline rs-fMRI (around 5 years prior to the conversion) to differentiate these two groups.

One major problem in this classification is imbalanced datasets. To deal with this problem, we have used a data augmentation method called adaptive synthetic (ADASYN) sampling approach. In this method, we adaptively generated synthetic data for the minatory class based on the distribution of both classes. ADASYN generates synthetic data for the part of minority class that is harder to learn than those minority samples, which are easier to learn. In this study, we have a dataset with 85 samples in major class and 40 samples in minor class. Using ADASYN, we generated 45 samples of synthetic data for the minor class to make the dataset balanced (He et al., 2008). We trained an SVM with polynomial kernel function, as shown in Eq. 2. to classify two classes (Cortes and Vapnic, 1995).

$$
k\left(x_{1}, x_{2}\right)=\left(1+x_{1}^{\prime} x_{2}\right)^{p}
$$

where $p$ is a positive integer value.

There are a few advantages of using SVM. (1) SVM works well for high dimensional data. (2) SVM is effective when the number of the sample is smaller than the number of dimensions, which is a common problem for neuroimaging data. (3) SVM can handle nonlinearity in the data using a kernel trick. This provides an advantage over linear classifiers like logistic regression. However, choosing the appropriate kernel function is not easy. In addition, due to the limited number of samples, it is not advisable to use a neural network classifier. It is worth mentioning that an imbalanced dataset is a challenging problem in SVM classification (Cervantes et al., 2020). Our study used ADASYN to generate synthetic data of the minatory class to elevate this problem. In more detail, we used the ADASYN-based sample and a subset of the major class (i.e., uc-HC, $N=45$, the ADASYN sample size) and trained a model and then we tested that model on real unseen data from both uc-HC and c-HC groups. We iterate this 10 times. This number was chosen arbitrary. It worth mentioning that changing the number of iterations would not change the classification result. In each iteration, we used five-fold crossvalidation method in which we used $80 \%$ of the training data to train a model and $20 \%$ of the data to validate that. The 
TABLE 2 | Component labels.

\begin{tabular}{|c|c|c|c|c|c|}
\hline & $\mathrm{SCN}$ & $\begin{array}{l}\text { Component name } \\
\text { Caudate (69) }\end{array}$ & \multicolumn{3}{|c|}{ Peak coordinate $(\mathrm{mm})$} \\
\hline 2 & & Subthalamus/hypothalamus (53) & -2.5 & -13.5 & -1.5 \\
\hline 4 & & Caudate (99) & 21.5 & 10.5 & -3.5 \\
\hline 5 & & Thalamus (45) & -12.5 & -18.5 & 11.5 \\
\hline 6 & ADN & Superior temporal gyrus ([STG], 21) & 62.5 & -22.5 & 7.5 \\
\hline 8 & SMN & Postcentral gyrus ([PoCG], 3) & 56.5 & -4.5 & 28.5 \\
\hline 9 & & Left postcentral gyrus ([L PoCG], 9) & -38.5 & -22.5 & 56.5 \\
\hline 10 & & Paracentral lobule ([ParaCL], 2) & 0.5 & -22.5 & 65.5 \\
\hline 11 & & Right postcentral gyrus ([R PoCG], 11) & 38.5 & -19.5 & 55.5 \\
\hline 12 & & Superior parietal lobule ([SPL], 27) & -18.5 & -43.5 & 65.5 \\
\hline 13 & & Paracentral lobule ([ParaCL], 54) & -18.5 & -9.5 & 56.5 \\
\hline 17 & & Calcarine gyrus ([CalcarineG], 16) & -12.5 & -66.5 & 8.5 \\
\hline 18 & & Middle occipital gyrus ([MOG], 5) & -23.5 & -93.5 & -0.5 \\
\hline 19 & & Middle temporal gyrus ([MTG], 62) & 48.5 & -60.5 & 10.5 \\
\hline 20 & & Cuneus (15) & 15.5 & -91.5 & 22.5 \\
\hline 21 & & Right middle occipital gyrus ([R MOG], 12) & 38.5 & -73.5 & 6.5 \\
\hline 22 & & Fusiform gyrus (93) & 29.5 & -42.5 & -12.5 \\
\hline 23 & & Inferior occipital gyrus ([IOG], 20) & -36.5 & -76.5 & -4.5 \\
\hline 24 & & Lingual gyrus ([LingualG], 8) & -8.5 & -81.5 & -4.5 \\
\hline 25 & & Middle temporal gyrus ([MTG], 77) & -44.5 & -57.5 & -7.5 \\
\hline 26 & $\mathrm{CCN}$ & Inferior parietal lobule ([IPL], 68) & 45.5 & -61.5 & 43.5 \\
\hline 27 & & Insula (33) & -30.5 & 22.5 & -3.5 \\
\hline 35 & & Superior frontal gyrus ([SFG], 96) & -24.5 & 26.5 & 49.5 \\
\hline 36 & & Middle frontal gyrus ([MiFG], 88) & 30.5 & 41.5 & 28.5 \\
\hline 37 & & Hippocampus ([HiPP], 48) & 23.5 & -9.5 & -16.5 \\
\hline 38 & & Left inferior parietal lobue ([L IPL], 81) & 45.5 & -61.5 & 43.5 \\
\hline 39 & & Middle cingulate cortex ([MCC], 37) & -15.5 & 20.5 & 37.5 \\
\hline 40 & & Inferior frontal gyrus ([IFG], 67) & 39.5 & 44.5 & -0.5 \\
\hline 41 & & Middle frontal gyrus ([MiFG], 38) & -26.5 & 47.5 & 5.5 \\
\hline 42 & & Hippocampus ([HiPP], 83) & -24.5 & -36.5 & 1.5 \\
\hline 43 & DMN & Precuneus (32) & -8.5 & -66.5 & 35.5 \\
\hline 44 & & Precuneus (40) & -12.5 & -54.5 & 14.5 \\
\hline 45 & & Anterior cingulate cortex ([ACC], 23) & -2.5 & 35.5 & 2.5 \\
\hline 46 & & Posterior cingulate cortex ([PCC], 71) & -5.5 & -28.5 & 26.5 \\
\hline 47 & & Anterior cingulate cortex $([\mathrm{ACC}], 17)$ & -9.5 & 46.5 & -10.5 \\
\hline 48 & & Precuneus (51) & -0.5 & -48.5 & 49.5 \\
\hline 49 & & Posterior cingulate cortex ([PCC], 94) & -2.5 & 54.5 & 31.5 \\
\hline 50 & CBN & Cerebellum ([CB], 13) & -30.5 & -54.5 & -42.5 \\
\hline 51 & & Cerebellum ([CB], 18) & -32.5 & -79.5 & -37.5 \\
\hline 52 & & Cerebellum ([CB], 4) & 20.5 & -48.5 & -40.5 \\
\hline 53 & & Cerebellum ([CB], 7) & 30.5 & -63.5 & -40.5 \\
\hline
\end{tabular}


hyperparameters of the SVM classifier were selected trough an optimization process.

We calculated the classification accuracy, sensitivity, specificity, and area under the receiver operating characteristic curve (AUC) to assess the classification performance. Accuracy, sensitivity, and specificity were quantified by:

$$
\begin{gathered}
\text { Accurcy }=\frac{T P+T N}{T P+F N+T N+F P} \\
\text { Sensitivity }=\frac{T P}{T P+F N} \\
\text { Specificity }=\frac{T N}{T N+F P}
\end{gathered}
$$

where TP, FN, TN, and FP denoted the number of uc-HC subjects correctly predicted, the number of c-HC subjects classified as uc-HC subject, the number of uc-HC correctly predicted, and the number of un-HC subjects classified as c-HC subject, respectively.

\section{RESULTS}

\section{Dynamic Functional Connectivity States}

Figure 3 shows the reoccurring connectivity states identified by the k-means clustering method. In all states, we observed strong positive connectivity within SMN and VSN, ad CBN. State 3 showed the strongest connectivity within SMN and within VSN among all states. In addition, this state had the highest connectivity between SMN and VSN. Also, this state was separated from other states by showing the lowest negative connectivity between SMN and VSN with the rest of the brain. State 1 showed the lowest connectivity between SMN and VSN. Finally, we measured the OCR of each subject in state1, state2, and state 3. OCR represents the amount of time each subject spends in each state. Results showed that subjects spent an average of $23.78,52.17$, and $24.05 \%$ in state 1 , state 2 , and state 3 , respectively.

\section{The Correlation Between State-Specific FNC and CDR-SOB}

Figure 4 showed the partial correlation between state-specific FNC and CDR-SOB while we controlled for age and gender. The significant correlations (uncorrected $p<0.05$ ) are shown in red (positive correlation) and blue (negative correlation). Also, a significant correlation that passes the multiple comparisons is marked by asterisks.

In state 1 , we observed a significant and negative correlation between within-SCN, within-SMN, within-VSN connectivity, and CDR-SOB. This means that this connectivity decreases by progression from normal to mild dementia states. In this state, we found a reorganized (i.e., both positive and negative) patterns in the correlation between within-DMN connectivity and within CCN-connectivity with CDR-SOB. A similar reorganized correlation pattern was observed between the between-network connection and CDR-SOB. Specifically, a more reorganized pattern was observed in the correlation between SCN connectivity with the rest of the brain.

In state 2 , similar to state 1 , we observed a negative correlation between the connectivity of within-SCN, withinSMN, within-VSN, within-CBN connectivity with CDR-SOB. We also observed both positive and negative correlations between the connectivity of CCN and DMN and CDR-SOB. Compared with the other states, this state showed a more significant correlation of within-CCN connectivity and CDR-SOB, in which many of them were positive. Also, within-DMN connectivity showed more negative connectivity compared with that of other states. This state also showed a positive correlation between connectivity between SMN and CBN and CDR-SOB. Also, we observed both positive and negative correlations between CDR-SOB and between-network connectivity in state 2 .

State 3 showed a significant and negative correlation between the within-SMN, within-VSN, within-DMN, within$\mathrm{CCN}$, and within-CBN connectivity and CDR-SOB. The amount of significant correlation between within-SMN, within-VSN, and between SMN and VSN connectivity with CDR-SOB was more than those of the other states. Also, this state showed a significant positive correlation between VSN and CBN connection and CDR-SOB. Overall, we observed a reorganized pattern in the correlation between CDR-SOB and betweennetwork connection in this state.

\section{The Correlation Between Temporal Properties of dFNC and CDR-SOB}

We calculated the partial correlation between CDR-SOB and temporal features of dFNC (i.e., OCR and HMM) by controlling the age and gender. We found a positive correlation between OCR of state 1 and CDR-SOB $(r=0.07$, corrected $p=0.009)$ and a negative correlation between OCR of state 3 and CDR-SOB $\left(r=-0.14\right.$, corrected $\left.p=2 \mathrm{e}^{-7}\right)$. Also, we observed a negative correlation between CDR-SOB and $\mathrm{a}_{11}$, i.e., the transition from state 1 to state $1(r=0.07$, corrected $p=0.02)$, and a positive correlation between CDR-SOB and $\mathrm{a}_{33}$, i.e., the transition within state $3(r=-0.11$, corrected $p=0.0001)$.

\section{Both Healthy and Patient Brain Follow Similar State Pattern}

Since the number of HC scan is more than the number of patient ones, we applied the clustering method to their dFNC of $\mathrm{HC}$ and patients, separately. The results are shown in Figure 5. This figure shows that our approach captured a similar brain state in both groups. We used the Pearson correlation between states' FNC to assess the similarity between them. The correlation between state 1 of $\mathrm{HC}$ with state 1 of the patient group, between state 2 of $\mathrm{HC}$ with state 2 of the patient group, and between state 3 of $\mathrm{HC}$ with state 3 of the patient group were $0.9903(N=1378$, $p<0.001), 0.9825(N=1378, p<0.001)$, and $0.9921(N=1378$, $p<0.001$ ), respectively (Figures 5A,B). In addition, the OCR followed a similar pattern with the results when we concatenated all subjects. State 2 shows the highest OCR among all three states in both groups. HC subjects have higher OCR in state 1 than 


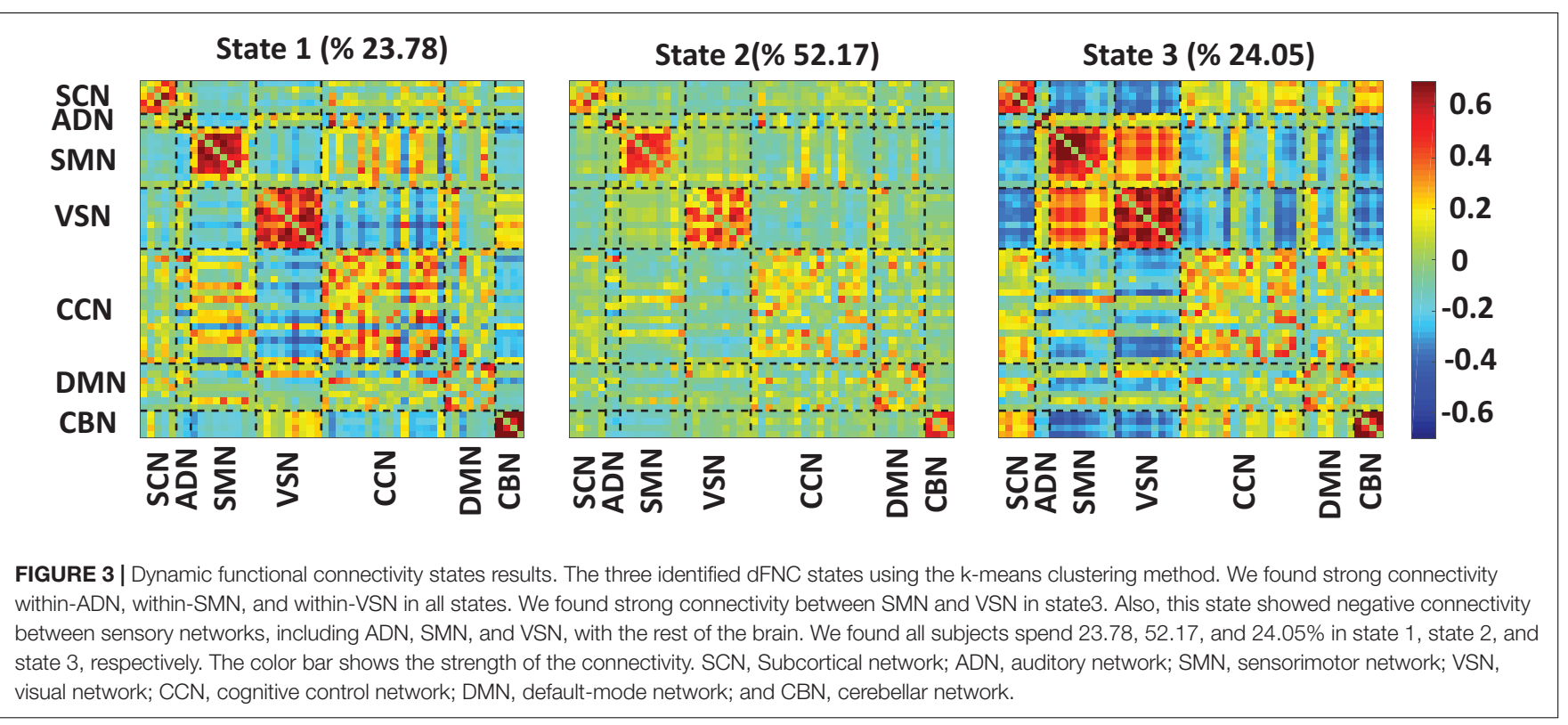

\begin{tabular}{l}
\hline State 1 \\
SMN
\end{tabular}

patients $(p<0.01)$, while patients have higher OCR in state 3 than that of HC subjects $(p<0.01)$ (Figures 5C,D). These results proved that the HC subject's dFNC did not dominate the state pattern. In addition, to reference the states easier in this paper, we put a name on each state. Since both healthy subjects and patients spend more than $50 \%$ of their scanning time in state 2 , we called this state a baseline. Since vmAD patients spend more time in state1, we called this state the vmAD-related state, and finally, we call state 3 , which healthy subjects spend more than in this state than state 2, as an HC-related state. Only in state 3, we observed a significant link between CDR-SOB and FNC after FDR correction (shown by asterisks). We observed a decrease in sensory network FNC by AD's progression. Also, we observed a reorganized pattern in the CCN FNC pattern.

\section{Changing the Number of States Does Not Change the Results}

To test whether the number of clusters (or states) would change the results or not, we applied the same clustering method for $k=5, k=7$, and $k=10$. Figures $6 \mathrm{~A}-\mathrm{C}$ show the results for $k=5$, $k=7$, and $k=10$, respectively. We observed similar state patterns with different $k$ values. State $1(k=3)$ is similar to state $1(k=5)$, state $2(k=7)$ and state $5(k=10)$. State $2(k=3)$ is similar to state 


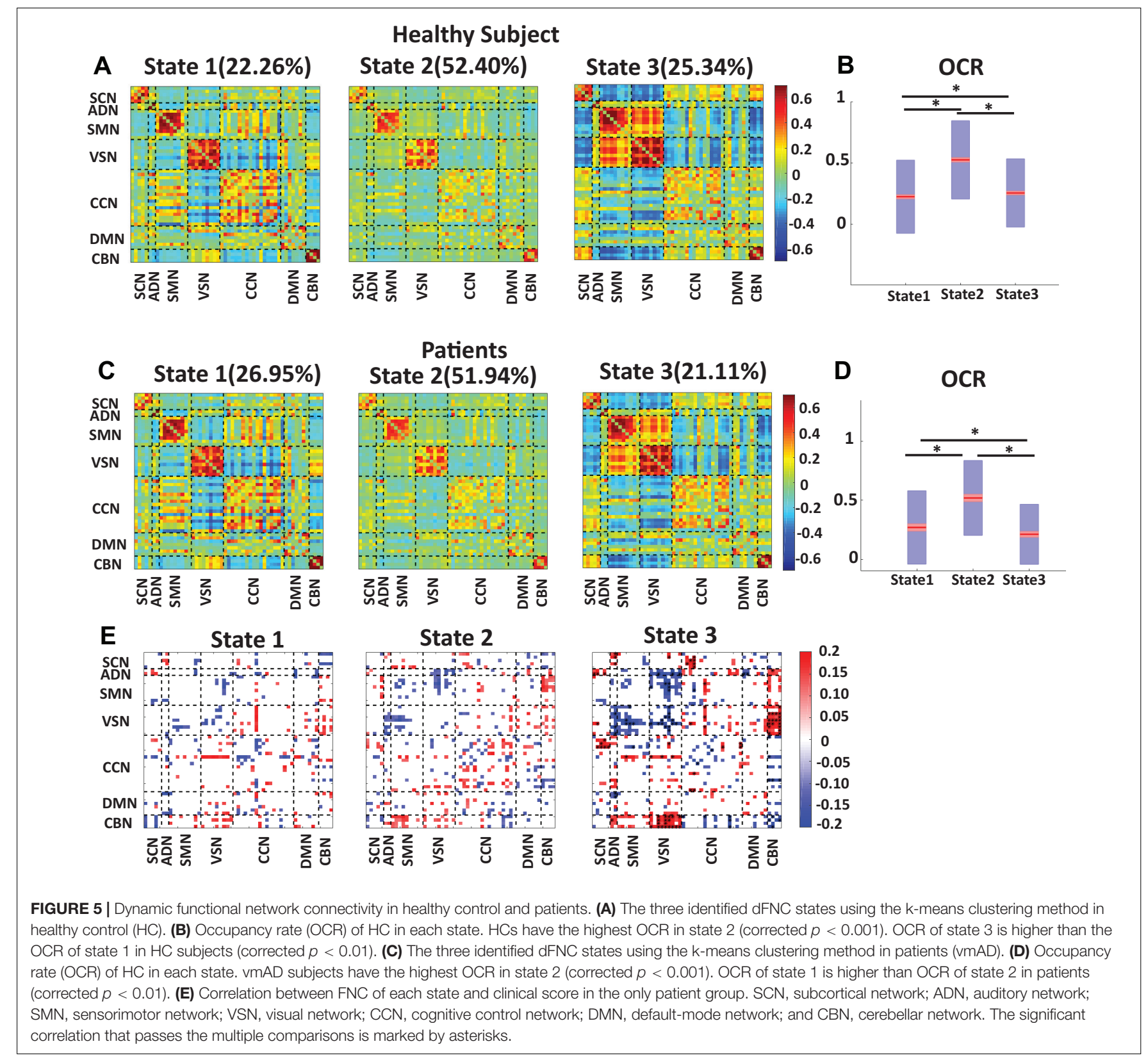

$2(k=5)$, state $1(k=7)$, and state $8(k=10)$. State $3(k=3)$ is similar to state $3(k=5)$, state $5(k=7)$, and state7 $(k=10)$. Increasing $\mathrm{k}$ above the optimized value of $k=3$ yields states whose similarity to those in the optimized value weakens as $\mathrm{k}$ grows. However, the two states (states 1 and 3) in the optimized clustering whose OCR is significantly linked to CDR-SOB are highly replicable up to $k=7$ and whose occupancy has a replicable significant relationship to CDR-SOB. Table 3 shows the correlation between CDR-SOB with OCR of clustering with different $k$ values.

\section{Dementia Progression Associated With Functional Network Connectivity}

Using baseline FNC features, including sFNC, HMM, and OCR, from whole-brain FNC, we successfully predicted the conversion from the normal state to vmAD by classifying those subjects converted to the mildly impaired stage (i.e., c-HC) from those stayed unchanged within 5 years, i.e., uc-HC. The average accuracy, sensitivity, specificity, and AUC in this classification was $75,72,78$, and $81 \%$, respectively.

\section{DISCUSSION}

In this study, we explored the dynamic of whole-brain FNC of $\mathrm{HC}(\mathrm{CDR}-\mathrm{SOB}=0)$ and vmAD $(0.5 \leq \mathrm{CDR}-\mathrm{SOB} \leq 9)$ subjects from the longitudinal rs-fMRI OASIS-3 (LaMontagne et al., 2019). Using a data-driven approach, we extracted 53 ICs for the whole brain and used a sliding window approach followed by 


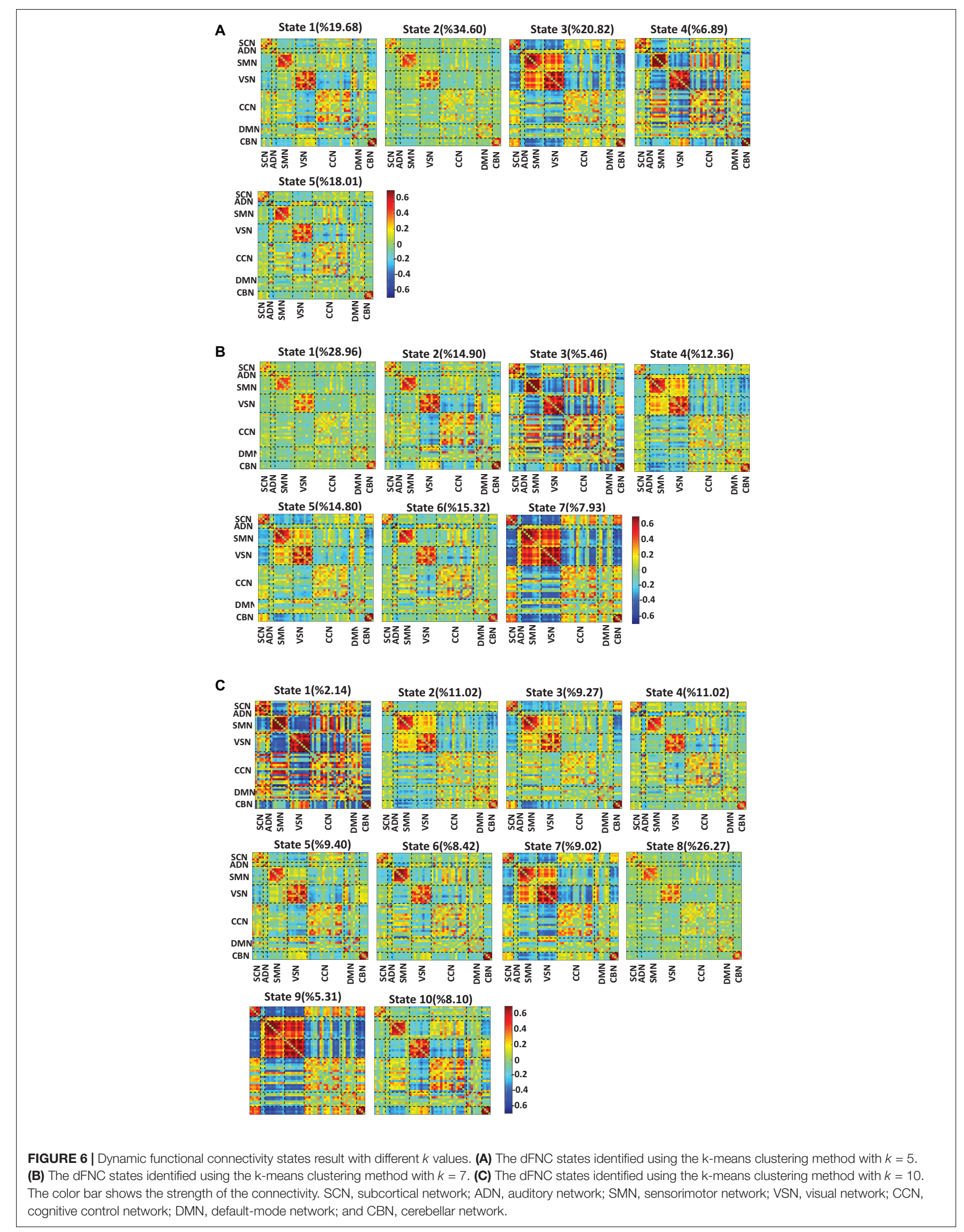


TABLE 3 | The link between occupancy rate of each state and CDR-SOB.

\begin{tabular}{|c|c|c|c|c|c|c|c|c|c|c|}
\hline & State1 & State2 & State3 & State4 & State5 & State6 & State7 & State8 & State9 & State10 \\
\hline \multirow[t]{2}{*}{$K=3$} & 0.07 & 0.02 & -0.14 & NA & NA & NA & NA & NA & NA & NA \\
\hline & $(0.013)$ & (0.09) & $(6 e-7)$ & & & & & & & \\
\hline \multirow[t]{2}{*}{$K=5$} & 0.088 & -0.014 & -0.083 & 0.033 & -0.017 & NA & NA & NA & NA & NA \\
\hline & $(0.001)$ & $(0.580)$ & $(0.001)$ & (0.026) & 0.513 & & & & & \\
\hline \multirow[t]{2}{*}{$K=7$} & $-3 e-4$ & 0.100 & -0.013 & -0.036 & -0.075 & 0.043 & -0.053 & NA & NA & NA \\
\hline & (0.991) & $(0.001)$ & $(0.722)$ & $(0.241)$ & $(0.017)$ & (0.188) & (0.106) & & & \\
\hline \multirow[t]{2}{*}{$K=10$} & $-7.8 e-4$ & -0.030 & -0.073 & 0.060 & 0.090 & -0.009 & -0.057 & -0.010 & -0.040 & 0.054 \\
\hline & $(0.771)$ & (0.368) & $(0.030)$ & $(0.080)$ & $(0.008)$ & $(0.771)$ & (0.082) & $(0.771)$ & (0.219) & (0.087) \\
\hline
\end{tabular}

The bold values are the significant correlation after multiple comparisons. All corrected $p$ values are in the parentheses.

a clustering method to study dFNC of this dataset. We found that the connectivity between VSN and SMN is dynamic by a transition from positive connectivity in state 3 to moderate positive connectivity in state 2 and negative connectivity in state 1 . In addition, the connectivity between SMN and VSN with the rest of the brain changes from negative connectivity in state 3 to other to state with more positive connectivity. Besides SMN and VSN, we found a dynamical pattern in CCN connectivity. Overall, we found the whole-brain FNC is highly dynamic. This result argues the previous $\mathrm{AD}$-related literature that mainly ignored the dynamic behavior of brain connectivity. Although a few studies explored the dFNC recently (Schumacher et al., 2019; Gu et al., 2020), the current study uses Neuromark as a replicable platform to extract data-driven ICs from relatively large longitudinal data. This replicable platform can generate ICs and replicate that across the different datasets. This would help reproduce similar brain states for FNC across different datasets, which is very important when studying dynamics (Iraji et al., 2020).

We found within-SMN FC decreased by the transition from a $\mathrm{HC}$ to a vmAD. This pattern was observed in all 3 states. Previous studies showed a decrease in SMN FC is shown in AD patients compared with that of HC subjects (Agosta et al., 2010; Damoiseaux et al., 2012; Wang P. et al., 2015; Li et al., 2020). In more detail, we found the FC within the postcentral gyrus decrease in vmAD than the HC brain. Postcentral gyrus has a key role in somatic sensation, including pain and temperature (DiGuiseppi and Tadi, 2019). Also, a previous study showed an impairment of pain and temperature sensation in mild dementia (Fletcher et al., 2015). Therefore, dysconnectivity in postcentral gyrus can potentially explain the impairment of somatic sensation in the early stage of dementia and suggest a prospective study.

In addition, we observed that the VSN FC, in the particular fusiform gyrus, decreases when the brain progress from the healthy state to mild dementia. As previous studies showed, the fusiform gyrus is involved in face recognition, and alteration in the connectivity between this brain region and other subregions of VSN causes impairment in face recognition (Fur et al., 2011; Cai et al., 2015). Functional dysconnectivity between fusiform and the rest of the VSN potentially can explain the impairment of face recognition in the early stage of $\mathrm{AD}$ progression (Uhlmann et al., 1991). Also, results indicated reduced FC among the brain sensory networks, i.e., $\mathrm{ADN}, \mathrm{SMN}$, and VSN, by progression from $\mathrm{HC}$ state to vmAD. Information processing integration of multisensory signals is a hallmark of self-awareness. For instance, (Ehrsson, 2007) showed that the matching between visual perception and proprioceptive signals is necessary for preserving the self-consciousness. Disconnection among sensory networks in mild dementia patients than that of healthy subjects can potentially explain the underlying mechanism of self-awareness discrepancy in $\mathrm{AD}$ patients. The current findings suggest future studies for exploring a causal link between dysconnectivity in the sensory network and lack of self-awareness in $\mathrm{AD}$ patients.

We also observed a disrupted temporal and spatial pattern in the connectivity between CBN and other brain networks. In all states, we found a decrease in the connectivity between CBN and $\mathrm{SCN}$ by advancement from the HC brain to vmAD. However, the connectivity between CBN and SMN, and between CBN and VSN are higher in vmAD than the HC subjects. This finding is consistent with a previous study that showed a reorganized pattern in the connectivity between cerebellar subregions and DMN, VSN, SMN (Zheng et al., 2017). However, we did not detect a significant pattern in the correlation between the clinical rate and the connectivity between CBN and DMN.

In addition, we found a disrupted pattern in DMN connectivity by having reduced connectivity in state 3 , and both increased and reduced connectivity in state 1 and state 2 for vmAD than the normal brain. Based on $\mathrm{sFC}$, previous studies reported both increase (Tao et al., 2017) and decrease (Binnewijzend et al., 2012) in whole-DMN connectivity of the AD subject. Another study reported no significant difference in DMN connectivity between AD patients with HC (Grieder et al., 2018). Although a small sample size might affect the statistical power, as previously shown in a study of major depression (Yan et al., 2019), this inconsistent result partially could be due to focusing on static FC, which is obtained from the correlation within an entire time series. Similarly, we observed a disrupted spatial and temporal pattern in CCN connectivity. In addition, we found a reorganized pattern in the connectivity between $\mathrm{CCN}$ and other networks such as SCN, ADN, SMN, VSN, DMN, and CBN. A recent study showed a reorganized pattern in the connectivity between inferior parietal lobule, as a part of $\mathrm{CCN}$, with default mode, salience, executive control, and SMNs (Wang Z. et al., 2015). Our new finding provides new knowledge about the reorganized pattern between $\mathrm{CNN}$ and the rest of the brain. The 
decrease in the CCN FC might explain the loss in the functional integrity of the CCN network, and the increased FC showed that vmAD patients potentially utilize additional brain subregions to compensate for the impairment of cognitive function. We also found that the FC within SCN, including caudate, thalamus, and putamen, decreased in vmAD patients compared with that of normal subjects. This result is consistent with a previous study that showed subjects with the risk of AD showed less connectivity in caudate and thalamus (Li W. et al., 2014).

Next, we calculated the correlation between clinical rate and OCR and HMM. We found HC subjects spend more time in state 3, which showed the highest positive connectivity among sensory networks, i.e., VSN, SMN, and ADN, is less in vmAD patients than the normal subjects. Also, we found the dwell time of state1, which showed the least connectivity among sensory networks, is higher for the patients with mild dementia. This finding provides further evidence of the effect of disease on the dysregulating temporal properties of FNC. A recent study showed that AD subjects spend more time in a sparsely connected state in which the motor network isolated from the rest of the brain (Schumacher et al., 2019). Our result is consistent with the study mentioned above by showing that the subjects with mild severity in the early stage of $\mathrm{AD}$ spend more time in state 1 , which shows sparse connectivity among brain networks. However, another part of our result that shows normal brain spend more time in state 3, in which SMN is isolated from most parts of the brain except VSN, contradicts the result of the aforementioned study. In addition, spending more time in a state with lower connectivity between SMN and VSN and less time in a state with stronger connectivity between SMN and VSN by subject in the early stage of $\mathrm{AD}$ emphasizes more on the role of this connectivity by the transition from the normal stage to the early stage of AD. Also, our result is consistent with another study from our group on a different dataset that showed that $\mathrm{AD}$ patients spend more time in the state with lower connectivity and spend less time in a state with higher connectivity (Du et al., 2019).

Prior work has demonstrated the regional patterns of $\mathrm{AD}$ pathology and their overlap with DMN regions (Desgranges et al., 2011). Therefore, we expected DMN to be impacted, as demonstrated in prior studies. However, we found that associations between primary sensory/motor networks were most correlative to symptom severity. Sensory and motor networks are considered relatively spared from $\mathrm{AD}$ pathology, at least until later stages of the disease. These exciting findings may suggest that although relatively preserved and potentially due to high signals in these regions, regions involved in crossmodal sensory/motor integration are damaged. This information provides a sensitive measure of neural damage in $\mathrm{AD}$ (potentially more sensitive than primary degeneration regions). Our result might suggest that DMN is the last brain network that is affected by $\mathrm{AD}$. Our result might also explain the previous study's finding that motor function changes might predate the cognitive impairments and dementia onset (Verghese et al., 2007; Wesson et al., 2011). However, a prospective study is needed to find which specific sensory or motor function changes sign early AD. Also, our new result about dysconnectivity in the somatosensory network might explain why physical exercise would prevent $\mathrm{AD}$
(De la Rosa et al., 2020) by increasing FNC among sensory networks (Demirakca et al., 2016).

Neurofeedback is a form of real-time biofeedback regulating brain activity and promoting brain function and behavioral performance (Omejc et al., 2019). In this technique, the neural signals are recorded from the brain. A feedback mechanism is then used to control the neural signal features through a feedback loop in the form of audio, video, or a combination of them. This closed-loop therapy has been widely used for major depressive disorder (Lee et al., 2019), attention deficit hyperactivity disorder (Enriquez-Geppert et al., 2019), and autism (Eroglu and Ekici, 2020) and got attention for treating $\mathrm{AD}$ in recent years. A recent study used the amount of delta, theta, alpha, and beta activity from EEG signal as a control signal in neurofeedback to improve cognitive function in AD (Luijmes et al., 2016). We introduced the sensory network's connectivity as a potential control marker in the neurofeedback in the current study. More specifically, our result suggests a possible benefit of administering the neurofeedback during the vmAD-related state and switching the brain state from vmAD-related to HCrelated state. Although many technical limitations of real-time implanting neurofeedback system integrated with dFNC exist (Monti et al., 2017; Watanabe et al., 2017). Our results suggest a future benefit of dFNC states in neurofeedback in AD treatment.

Finally, we show that both dFNC and sFNC can be used to predict the conversion from healthy to vmAD based on their baseline recording. Previous literature proposed a few models to predict conversion from MCI to AD (Risacher et al., 2009; Li H. et al., 2014; Abrol et al., 2020). For example, one study used 75 state $\mathrm{MCI}$, i.e., who did not convert to $\mathrm{AD}$, and 51 progressive MCI, i.e., who changed to AD within 3 years, modeled a SVM and could classify them with $79.37 \%$ accuracy based on the brain connectivity features (Zheng et al., 2019). Another study used structural and genetic data for prediction from converted normal subjects to mild cognitive impairment from the unconverted normal subject within 5 years and could predict the conversion from normal to mild cognitive impairment with AUC of $85 \%$ (Albert et al., 2018). However, the model for the conversion from the normal brain to mild impairment state based on their baseline recording has not extensively reported. The current study shows a potential for FNC in predicting from healthy aging to mild impairment stage.

\section{Limitations and Future Study}

There are some limitations to this work. The choice of window size is an implicit assumption about the dynamic behavior in that a short window captures more rapid fluctuations, whereas a longer window does more smoothing. Future work can be accomplished to evaluate the range of dynamics more comprehensively (Faghiri et al., 2020). In addition, we used SVM to compute the classification between individuals who converted to $\mathrm{vmAD}$, and those did not convert. Other more advanced methods like neural network-based classification can potentially increase the prediction accuracy. However, applying neural network-based classification is almost impossible due to the limited number of samples in the longitudinal data used in this classification. 


\section{CONCLUSION}

In work reported here, we extend this existing body of knowledge into the dynamic realm, investigating how timevarying properties of whole-brain FNC changes by the transition from healthy aging to $\mathrm{vmAD}$. We found a state-specific reorganized pattern in the whole-brain FNC of vmAD patients. We observed a decreased connectivity among sensory networks, including SMN, VSN, and ADN, in mild dementia state. This provides a piece of new knowledge about the sensory network dysconnectivity in the early stage of $\mathrm{AD}$ with mild symptom severity. This potentially marked that sensory network is one of the brain networks that got affected more than the other brain network in the early stage of AD. In addition, we found a reorganized pattern, i.e., both increase and decrease in DMN and CCN connectivity. A similar changed pattern was observed in between-networks connectivity. We also found that mild dementia is linked to the temporal pattern on FNC by increasing the amount of the time staying in a sparsely connected state with lower functional connectivity among sensory networks. These results emphasized that not only the transition from the normal state to mild dementia changes the connectivity strength, but also it dysregulates the temporal properties of FNC.

\section{DATA AND CODE AVAILABILITY STATEMENT}

The datasets for this study from Open Access Series of Imaging Studies (OASIS)-3 (https://www.oasis-brains.org/). The preprocessed data are available from the corresponding author upon reasonable request. The code used for preprocessing and dFNC calculation are available at https://trendscenter.org/ software/. For generating the synthetic data, we used the code provided by Dominic Siedhoff (2020). ADASYN (improves class balance, an extension of SMOTE) (https://www.mathworks. com/matlabcentral/fileexchange/50541-adasyn-improves-classbalance-extension-of-smote).

\section{REFERENCES}

Abrol, A., Bhattarai, M., Fedorov, A., Du, Y., Plis, S., and Calhoun, V. (2020). Deep residual learning for neuroimaging: an application to predict progression to Alzheimer's disease. J. Neurosci. Methods 339, 108701. doi: 10.1016/j.jneumeth. 2020.108701

Abrol, A., Rashid, B., Rachakonda, S., Damaraju, E., and Calhoun, V. D. (2017). Schizophrenia shows disrupted links between brain volume and dynamic functional connectivity. Front. Neurosci. 11:624. doi: 10.3389/fnins.2017. 00624

Agosta, F., Rocca, M. A., Pagani, E., Absinta, M., Magnani, G., Marcone, A., et al. (2010). Sensorimotor network rewiring in mild cognitive impairment and Alzheimer's disease. Hum. Brain Mapp. 31, 515-525. doi: 10.1002/hbm. 20883

Albert, M., Zhu, Y., Moghekar, A., Mori, S., Miller, M. I., Soldan, A., et al. (2018). Predicting progression from normal cognition to mild cognitive impairment for individuals at 5 years. Brain 141, 877-887. doi: 10.1093/brain/awx365

Allen, E. A., Damaraju, E., Plis, S. M., Erhardt, E. B., Eichele, T., and Calhoun, V. D. (2014). Tracking whole-brain connectivity dynamics in the resting state. Cereb. Cortex 24, 663-676. doi: 10.1093/cercor/bhs352

\section{ETHICS STATEMENT}

The studies involving human participants were reviewed and approved by in this study, ethical approval was granted by the relevant Ethics Committees, and informed consent was obtained from each subject prior to scanning according to the Institutional Review Board of Washington University School of Medicine. The patients/participants provided their written informed consent to participate in this study.

\section{AUTHOR CONTRIBUTIONS}

MS developed the study, conducted data analysis, interpreted the results, and wrote the original manuscript draft. EZ conducted data analysis and wrote the manuscript draft. ZF and YD conducted data analysis and edited the original draft. JL, $\mathrm{RM}, \mathrm{EM}$, and DS edited the original draft and provided a critical review of the initial draft. VC developed the study, interpreted the results, edited the original draft, and provided critical review to the initial draft. All authors approved the final manuscript.

\section{FUNDING}

This work was supported by the NIH grants funded this work: R01AG063153, R01EB020407, R01MH094524, R01MH119069, R01MH118695, and R01MH121101. This work was also supported by National Natural Science Foundation of China (Grant No. 61703253 to YD).

\section{ACKNOWLEDGMENTS}

We thank those who helped collect this valuable data.

Benjamini, Y., and Hochberg, Y. (1995). Controlling the false discovery rate: a practical and powerful approach to multiple testing. R. Stat. Soc. Ser. B 57, 289-300. doi: 10.1111/j.2517-6161.1995.tb02031.x

Binnewijzend, M. A. A., Schoonheim, M. M., Sanz-Arigita, E., Wink, A. M., van der Flier, W. M., Tolboom, N., et al. (2012). Resting-state fMRI changes in Alzheimer's disease and mild cognitive impairment. Neurobiol. Aging 33, 2018-2028. doi: 10.1016/j.neurobiolaging.2011.07.003

Brier, M. R., Thomas, J. B., and Ances, B. M. (2014). Network dysfunction in Alzheimer's disease: refining the disconnection hypothesis. Brain Connect. 4, 299-311. doi: 10.1089/brain.2014.0236

Cai, S., Chong, T., Zhang, Y., Li, J., von Deneen, K. M., Ren, J., et al. (2015). Altered functional connectivity of fusiform gyrus in subjects with amnestic mild cognitive impairment: A resting-state fMRI study. Front. Hum. Neurosci. 9:471. doi: 10.3389/fnhum.2015.00471

Calhoun, V. D., Miller, R., Pearlson, G., and Adali, T. (2014). The chronnectome: time-varying connectivity networks as the next frontier in fMRI data discovery. Neuron 84, 262-274. doi: 10.1016/j.neuron.2014. 10.015

Cerliani, L., Mennes, M., Thomas, R. M., Di Martino, A., Thioux, M., and Keysers, C. (2015). Increased functional connectivity between subcortical and cortical 
resting-state networks in Autism spectrum disorder. JAMA Psychiatry 72, 767-777. doi: 10.1001/jamapsychiatry.2015.0101

Cervantes, J., Garcia-Lamont, F., Rodríguez-Mazahua, L., and Lopez, A. (2020). A comprehensive survey on support vector machine classification: applications, challenges and trends. Neurocomputing 408, 189-215. doi: 10.1016/j.neucom. 2019.10.118

Cortes, C., and Vapnic, V. (1995). Support-vector networks. Mach. Learn. 20, 273-297.

Damaraju, E., Allen, E. A., Belger, A., Ford, J. M., McEwen, S., Mathalon, D. H., et al. (2014). Dynamic functional connectivity analysis reveals transient states of dysconnectivity in schizophrenia. NeuroImage 5, 298-308. doi: 10.1016/j.nicl. 2014.07.003

Damoiseaux, J. S., Prater, K. E., Miller, B. L., and Greicius, M. D. (2012). Functional connectivity tracks clinical deterioration in Alzheimer's disease. Neurobiol. Aging 33, 828.e19-e30. doi: 10.1016/j.neurobiolaging.2011.06.024

De la Rosa, A., Olaso-Gonzalez, G., Arc-Chagnaud, C., Millan, F., Salvador-Pascual, A., García-Lucerga, C., et al. (2020). Physical exercise in the prevention and treatment of Alzheimer's disease. J. Sport Health Sci. 9, 394-404. doi: 10.1016/j. jshs.2020.01.004

de Lacy, N., Doherty, D., King, B. H., Rachakonda, S., and Calhoun, V. D. (2017). Disruption to control network function correlates with altered dynamic connectivity in the wider autism spectrum. NeuroImage 15, 513-524. doi: 10. 1016/j.nicl.2017.05.024

Demirakca, T., Cardinale, V., Dehn, S., Ruf, M., and Ende, G. (2016). The exercising brain: changes in functional connectivity induced by an integrated multimodal cognitive and whole-body coordination training. Neural Plast. 2016, 8240894. doi: 10.1155/2016/8240894

Desgranges, B., Mevel, K., Chételat, G., and Eustache, F. (2011). The default mode network in healthy aging and Alzheimer's disease. Int. J. Alzheimers Dis. 2011, 535816. doi: 10.4061/2011/535816

DiGuiseppi, J., and Tadi, P. (2019). Neuroanatomy, Postcentral Gyrus. Treasure Island, FL: StatPearls.

Du, Y., Fu, Z., Sui, J., Gao, S., Xing, Y., Lin, D., et al. (2019). NeuroMark: an adaptive independent component analysis framework for estimating reproducible and comparable fMRI biomarkers among brain disorders. medRxiv[Preprint] doi: $10.1101 / 19008631$

Du, Y., Fu, Z., Sui, J., Gao, S., Xing, Y., Lin, D., et al. (2020). NeuroMark: an automated and adaptive ICA based pipeline to identify reproducible fMRI markers of brain disorders. NeuroImage 28, 102375. doi: 10.1016/j.nicl.2020. 102375

Ehrsson, H. H. (2007). The experimental induction of out-of-body experiences. Science 317, 1048. doi: 10.1126/science.1142175

Enriquez-Geppert, S., Smit, D., Pimenta, M. G., and Arns, M. (2019). Neurofeedback as a treatment intervention in ADHD: current evidence and practice. Curr. Psychiatry Rep. 21, 46. doi: 10.1007/s11920-019-1021-4

Eroglu, G., and Ekici, B. (2020). Neurofeedback improves EEG complexity and social interaction in a boy with autism: a case report. J. Surg. Med. 4, 4-6. doi: 10.28982/josam.638667

Faghiri, A., Iraji, A., Damaraju, E., Turner, J., and Calhoun, V. D. (2020). A unified approach for characterizing static/dynamic connectivity frequency profiles using filter banks. Network Neurosci. doi: 10.1162/netn_a_00155 [Epub ahead of print].

Fiorenzato, E., Strafella, A. P., Kim, J., Schifano, R., Weis, L., Antonini, A., et al. (2019). Dynamic functional connectivity changes associated with dementia in Parkinson's disease. Brain 142, 2860-2872. doi: 10.1093/brain/awz192

Fletcher, P. D., Downey, L. E., Golden, H. L., Clark, C. N., Slattery, C. F., Paterson, R. W., et al. (2015). Pain and temperature processing in dementia: a clinical and neuroanatomical analysis. Brain 138, 3360-3372. doi: 10.1093/brain/awv276

Fu, Z., Caprihan, A., Chen, J., Du, Y., Adair, J. C., Sui, J., et al. (2019). Altered static and dynamic functional network connectivity in Alzheimer's disease and subcortical ischemic vascular disease: shared and specific brain connectivity abnormalities. Hum. Brain Mapp. 40, 3203-3221. doi: 10.1002/hbm.24591

Fu, Z., Iraji, A., Turner, J. A., Sui, J., Miller, R., Pearlson, G. D., et al. (2021). Dynamic state with covarying brain activity-connectivity: on the pathophysiology of schizophrenia. NeuroImage 224, 117385. doi: 10.1016/j. neuroimage.2020.117385

Fu, Z., Sui, J., Turner, J. A., Du, Y., Assaf, M., Pearlson, G. D., et al. (2020). Dynamic functional network reconfiguration underlying the pathophysiology of schizophrenia and autism spectrum disorder. Hum. Brain Mapp. 42, 80-94. doi: 10.1002/hbm.25205

Fur, N., Garrido, L., Dolan, R. J., Driver, J., and Duchaine, B. (2011). Fusiform gyrus face selectivity relates to individual differences in facial recognition ability. J. Cogn. Neurosci. 23, 1723-1740. doi: 10.1162/jocn.2010. 21545

Grieder, M., Wang, D. J. J., Dierks, T., Wahlund, L.-O., and Jann, K. (2018). Default mode network complexity and cognitive decline in mild Alzheimer's disease. Front. Neurosci. 12:770. doi: 10.3389/fnins.2018.00770

Gu, Y., Lin, Y., Huang, L., Ma, J., Zhang, J., Xiao, Y., et al. (2020). Abnormal dynamic functional connectivity in Alzheimer's disease. CNS Neurosci. Therap. 26, 962-971. doi: 10.1111/cns.13387

He, H., Bai, Y., Garcia, E., and Li, S. (2008). "ADASYN: adaptive synthetic sampling approach for imbalanced learning," in IEEE International Joint Conference on Neural Networks, 2008. IJCNN 2008.(IEEE World Congress on Computational Intelligence), (Hong Kong: IEEE), 1322-1328.

Iraji, A., Miller, R., Adali, T., and Calhoun, V. D. (2020). Space: a missing piece of the dynamic puzzle. Trends Cogn. Sci. 24, 135-149. doi: 10.1016/j.tics.2019.12. 004

LaMontagne, P. J., Benzinger, T. L. S., Morris, J. C., Keefe, S., Hornbeck, R., Xiong, C., et al. (2019). OASIS-3: longitudinal neuroimaging, clinical, and cognitive dataset for normal aging and Alzheimer Disease. medRxiv doi: 10.1101/2019. 12.13.19014902

Lee, Y. J., Lee, G. W., Seo, W. S., Koo, B. H., Kim, H. G., and Cheon, E. J. (2019). Neurofeedback treatment on depressive symptoms and functional recovery in treatment-resistant patients with major depressive disorder: an open-label pilot study. J. Korean Med. Sci. 34, 1-16. doi: 10.3346/jkms.2019.34.e287

Li, H., Liu, Y., Gong, P., Zhang, C., and Ye, J. (2014). Hierarchical interactions model for predicting Mild cognitive impairment (MCI) to Alzheimer's disease (AD) conversion. PLoS One 9:e82450. doi: 10.1371/journal.pone.008 2450

Li, W., Antuono, P. G., Xie, C., Chen, G., Jones, J. L., Ward, B. D., et al. (2014). Aberrant functional connectivity in Papez circuit correlates with memory performance in cognitively intact middle-aged APOE4 carriers. Cortex 57, 167-176. doi: 10.1016/j.cortex.2014.04.006

Li, W., Wen, W., Chen, X., Ni, B., Lin, X., and Fan, W. (2020). Functional evolving patterns of cortical networks in progression of Alzheimer' s disease: a graph-based resting-state fMRI study. Neural Plast. 2020, 7839536.

Luijmes, R. E., Pouwels, S., and Boonman, J. (2016). The effectiveness of neurofeedback on cognitive functioning in patients with Alzheimer's disease: preliminary results. Neurophysiol. Clin. 46, 179-187. doi: 10.1016/j.neucli.2016. 05.069

Mash, L. E., Linke, A. C., Olson, L. A., Fishman, I., Liu, T. T., and Müller, R. A. (2019). Transient states of network connectivity are atypical in autism: a dynamic functional connectivity study. Hum. Brain Mapp. 40, 2377-2389. doi: 10.1002/hbm.24529

Masters, C. L., Bateman, R., Blennow, K., Rowe, C. C., Sperling, R. A., and Cummings, J. L. (2015). Alzheimer's disease. Nat. Rev. Dis. Prim. 1, 1-18. doi: 10.1038/nrdp.2015.56

Monti, R. P., Lorenz, R., Braga, R. M., Anagnostopoulos, C., Leech, R., and Montana, G. (2017). Real-time estimation of dynamic functional connectivity network. Hum. Brain Mapp. 38, 202-220. doi: 10.1002/hbm. 23355

O’Bryant, S. E., Waring, S. C., Cullum, C. M., Hall, J., Lacritz, L., Massman, P. J., et al. (2008). Staging dementia using clinical dementia rating scale sum of boxes scores: a Texas Alzheimer's research consortium study. Arch. Neurol. 65, 1091-1095. doi: 10.1001/archneur.65.8.1091

Omejc, N., Rojc, B., Battaglini, P. P., and Marusic, U. (2019). Review of the therapeutic neurofeedback method using electroencephalography: EEG neurofeedback. Bosnian J. Basic Med. Sci. 19, 213-220. doi: 10.17305/bjbms. 2018.3785

Preti, M. G., Bolton, T. A., and Van De Ville, D. (2017). The dynamic functional connectome: state-of-the-art and perspectives. NeuroImage 160, 41-54. doi: 10.1016/j.neuroimage.2016.12.061

Risacher, S. L., Saykin, A. J., West, J. D., Shen, L., Firpi, H. A., Mcdonald, B. C., et al. (2009). Baseline MRI predictors of conversion from MCI to probable $\mathrm{AD}$ in the ADNI Cohort. Curr. Alzheimer Res. 6, 347-361. doi: 10.2174/ 156720509788929273 
Ryan, N. S., and Rossor, M. N. (2011). Defining and describing the pre-dementia stages of familial Alzheimer's disease. Alzheimers Res. Ther. 3, 2010-2012. doi: 10.1186/alzrt91

Schumacher, J., Peraza, L. R., Firbank, M., Thomas, A. J., Kaiser, M., Gallagher, P., et al. (2019). Dynamic functional connectivity changes in dementia with Lewy bodies and Alzheimer's disease. NeuroImage 22, 101812. doi: 10.1016/j. nicl.2019.101812

Sendi, M. S. E., Zendehrouh, E., Fu, Z., Mahmoudi, B., Miller, R. L., and Calhoun, V. D. (2020). "A machine learning model for exploring aberrant functional network connectivity transition in schizophrenia," in Proceedings of the IEEE Southwest Symposium on Image Analysis and Interpretation 2020March, (Albuquerque, NM: IEEE), 112-115. doi: 10.1109/SSIAI49293.2020. 9094620

Soman, S. M., Raghavan, S., Rajesh, P. G., Mohanan, N., Thomas, B., Kesavadas, C., et al. (2020). Does resting state functional connectivity differ between mild cognitive impairment and early Alzheimer's dementia? J. Neurol. Sci. 418, 117093. doi: 10.1016/j.jns.2020.117093

Tao, W., Li, X., Zhang, J., Chen, Y., Ma, C., Liu, Z., et al. (2017). Inflection point in course of mild cognitive impairment: increased functional connectivity of default mode network. J. Alzheimers Dis. 60, 679-690. doi: 10.3233/JAD170252

Uhlmann, R. F., Larson, E. B., Koepsell, T. D., Rees, T. S., and Duckert, L. G. (1991). Visual impairment and cognitive dysfunction in Alzheimer's disease. J. Gen. Intern. Med. 6, 126-132. doi: 10.1007/BF02598307

Verghese, J., Wang, C., Lipton, R. B., Holtzer, R., and Xue, X. (2007). Quantitative gait dysfunction and risk of cognitive decline and dementia. J. Neurol. Neurosurg. Psychiatry 78, 929-935. doi: 10.1136/jnnp.2006.106914

Wang, P., Zhou, B., Yao, H., Zhan, Y., Zhang, Z., Cui, Y., et al. (2015). Aberrant intra-and inter-network connectivity architectures in Alzheimer's disease and mild cognitive impairment. Sci. Rep. 5, 1-12. doi: 10.1038/srep14824

Wang, X. H., Jiao, Y., and Li, L. (2018). Identifying individuals with attention deficit hyperactivity disorder based on temporal variability of dynamic functional connectivity. Sci. Rep. 8, 1-12. doi: 10.1038/s41598-018-30308-w

Wang, Z., Xia, M., Dai, Z., Liang, X., Song, H., He, Y., et al. (2015). Differentially disrupted functional connectivity of the subregions of the inferior parietal lobule in Alzheimer's disease. Brain Struct. Funct. 220, 745-762. doi: 10.1007/ s00429-013-0681-9

Watanabe, T., Sasaki, Y., Shibata, K., and Kawato, M. (2017). Advances in fMRI real-time neurofeedback. Trends Cogn. Sci. 21, 997-1010. doi: 10.1016/j.tics. 2017.09.010

Wesson, D. W., Borkowski, A. H., Landreth, G. E., Nixon, R. A., Levy, E., and Wilson, D. A. (2011). Sensory network dysfunction, behavioral impairments, and their reversibility in an alzheimer's $\beta$-amyloidosis mouse model. J. Neurosci. 31, 15962-15971. doi: 10.1523/JNEUROSCI.2085-11. 2011

Yan, C. G., Chen, X., Li, L., Castellanos, F. X., Bai, T. J., Bo, Q. J., et al. (2019). Reduced default mode network functional connectivity in patients with recurrent major depressive disorder. Proc. Natl. Acad. Sci. U.S.A. 116, 9078-9083. doi: 10.1073/pnas.1900390116

Yiannopoulou, K. G., and Papageorgiou, S. G. (2020). Current and future treatments in Alzheimer disease: an update. J. Cent. Nerv. Syst. Dis. 12, 117957352090739. doi: 10.1177/1179573520907397

Zendehrouh, E., Sendi, M. S. E., Sui, J., Fu, Z., Zhi, D., Lv, L., et al. (2020). "Aberrant functional network connectivity transition probability in major depressive disorder," 42nd Annual International Conference of the IEEE Engineering in Medicine \& Biology Society (EMBC), Piscataway, NJ.

Zheng, W., Liu, X., Song, H., Li, K., and Wang, Z. (2017). Altered functional connectivity of cognitive-related cerebellar Subregions in Alzheimer's disease. Front. Aging Neurosci. 9:143. doi: 10.3389/fnagi.2017. 00143

Zheng, W., Yao, Z., Li, Y., Zhang, Y., Hu, B., and Wu, D. (2019). Brain connectivity based prediction of Alzheimer's disease in patients with mild cognitive impairment based on multi-modal images. Front. Hum. Neurosci. 13:399. doi: 10.3389/fnhum.2019.00399

Zhi, D., Calhoun, V. D., Lv, L., Ma, X., Ke, Q., Fu, Z., et al. (2018). Aberrant dynamic functional network connectivity and graph properties in major depressive disorder. Front. Psychiatry 9:339. doi: 10.3389/fpsyt.2018. 00339

Conflict of Interest: The authors declare that the research was conducted in the absence of any commercial or financial relationships that could be construed as a potential conflict of interest.

The reviewers SK and AC-P declared a shared affiliation with several of the authors, MS and VC, respectively, to the handling editor at the time of review.

Copyright (C) 2021 Sendi, Zendehrouh, Miller, Fu, Du, Liu, Mormino, Salat and Calhoun. This is an open-access article distributed under the terms of the Creative Commons Attribution License (CC BY). The use, distribution or reproduction in other forums is permitted, provided the original author(s) and the copyright owner(s) are credited and that the original publication in this journal is cited, in accordance with accepted academic practice. No use, distribution or reproduction is permitted which does not comply with these terms. 